\title{
Adaptive Resource Allocation in Multiuser OFDM Systems With Proportional Rate Constraints
}

\author{
Zukang Shen, Student Member, IEEE, Jeffrey G. Andrews, Member, IEEE, \\ and Brian L. Evans, Senior Member, IEEE
}

\begin{abstract}
Multiuser orthogonal frequency division multiplexing (MU-OFDM) is a promising technique for achieving high downlink capacities in future cellular and wireless local area network (LAN) systems. The sum capacity of MU-OFDM is maximized when each subchannel is assigned to the user with the best channel-to-noise ratio for that subchannel, with power subsequently distributed by water-filling. However, fairness among the users cannot generally be achieved with such a scheme. In this paper, a set of proportional fairness constraints is imposed to assure that each user can achieve a required data rate, as in a system with quality of service guarantees. Since the optimal solution to the constrained fairness problem is extremely computationally complex to obtain, a low-complexity suboptimal algorithm that separates subchannel allocation and power allocation is proposed. In the proposed algorithm, subchannel allocation is first performed by assuming an equal power distribution. An optimal power allocation algorithm then maximizes the sum capacity while maintaining proportional fairness. The proposed algorithm is shown to achieve about $95 \%$ of the optimal capacity in a twouser system, while reducing the complexity from exponential to linear in the number of subchannels. It is also shown that with the proposed resource allocation algorithm, the sum capacity is distributed more fairly and flexibly among users than the sum capacity maximization method.
\end{abstract}

Index Terms-Channel capacity, dynamic resource allocation, multiuser OFDM, proportional fairness, water-filling.

\section{INTRODUCTION}

$\mathbf{O}$ RTHOGONAL frequency division multiplexing (OFDM) is a promising technique for the next generation of wireless communication systems [1], [2]. OFDM divides the available bandwidth into $N$ orthogonal subchannels. By adding a cyclic prefix (CP) to each OFDM symbol, the channel appears to be circular if the $\mathrm{CP}$ length is longer than the channel length. Each subchannel can, thus, be modeled as a time-varying gain plus additive white Gaussian noise (AWGN). Besides the improved immunity to fast fading [3] brought by the multicarrier property of OFDM systems, multiple access is also possible, because the subchannels are orthogonal to each other.

Multiuser OFDM (MU-OFDM) adds multiple access to OFDM by allowing a number of users to share an OFDM sym-

Manuscript received June 16, 2003; revised June 15, 2004; accepted September 24, 2004. The editor coordinating the review of this paper and approving it for publication is L.-C. Wang. The work of B. L. Evans was supported by the State of Texas Advanced Technology Program under Grant 003658-0614-2001.

The authors are with the Wireless Networking and Communications Group, Department of Electrical and Computer Engineering, The University of Texas at Austin, Austin, TX 78712 USA (e-mail: shen@ece.utexas.edu; jandrews@ece.utexas.edu; bevans@ece.utexas.edu).

Digital Object Identifier 10.1109/TWC.2005.858010 bol. Two classes of resource allocation schemes exist, namely: 1) fixed resource allocation [4]; and 2) dynamic resource allocation [5]-[8]. Fixed resource allocation schemes, such as time division multiple access (TDMA) and frequency division multiple access (FDMA), assign an independent dimension, e.g., time slot or subchannel, to each user. A fixed resource allocation scheme is not optimal, since the scheme is fixed regardless of the current channel condition. On the other hand, dynamic resource allocation allocates a dimension adaptively to the users based on their channel gains. Due to the time-varying nature of the wireless channel, dynamic resource allocation makes full use of multiuser diversity to achieve higher performance.

Two classes of optimization techniques have been proposed in the dynamic multiuser OFDM literature, namely: 1) margin adaptive (MA) [5]; and 2) rate adaptive (RA) [6], [7]. The MA objective is to achieve the minimum overall transmit power given the constraints on the users' data rates or bit error rates (BER). The RA objective is to maximize each user's errorfree capacity with a total transmit power constraint. These optimization problems are nonlinear and, hence, computationally intensive to solve. In [8], the nonlinear optimization problems were transformed into a linear optimization problem with integer variables. The optimal solution can be achieved by integer programming. However, even with integer programming, the complexity increases exponentially with the number of constraints and variables.

Two RA optimization problems have been proposed by researchers. Recently, Jang and Lee proposed the rate maximization problem [6]. In [6], they proved that the sum capacity is maximized when each subchannel is assigned to the user with the best subchannel gain and power is then distributed by the water-filling algorithm. However, fairness is not considered in [6]. When the path loss differences among users are large, it is possible that the users with higher average channel gains will be allocated most of the resources, i.e., subchannels and power, for a significant portion of time. The users with lower average channel gains may be unable to receive any data, since most of the time the subchannels will be assigned to users with higher channel gains. In [7], Rhee and Cioffi studied the max-min problem, where by maximizing the worst user's capacity, it is assured that all users achieve a similar data rate. However, the max-min optimization problem can only provide maximum fairness among the users. In most wireless systems of interest, different users require different data rates, which may be accommodated by allowing users to subscribe to different levels of service.

In [9], Viswanath et al. discussed long-term proportional fairness resource allocation with "dumb" antennas. They pointed 


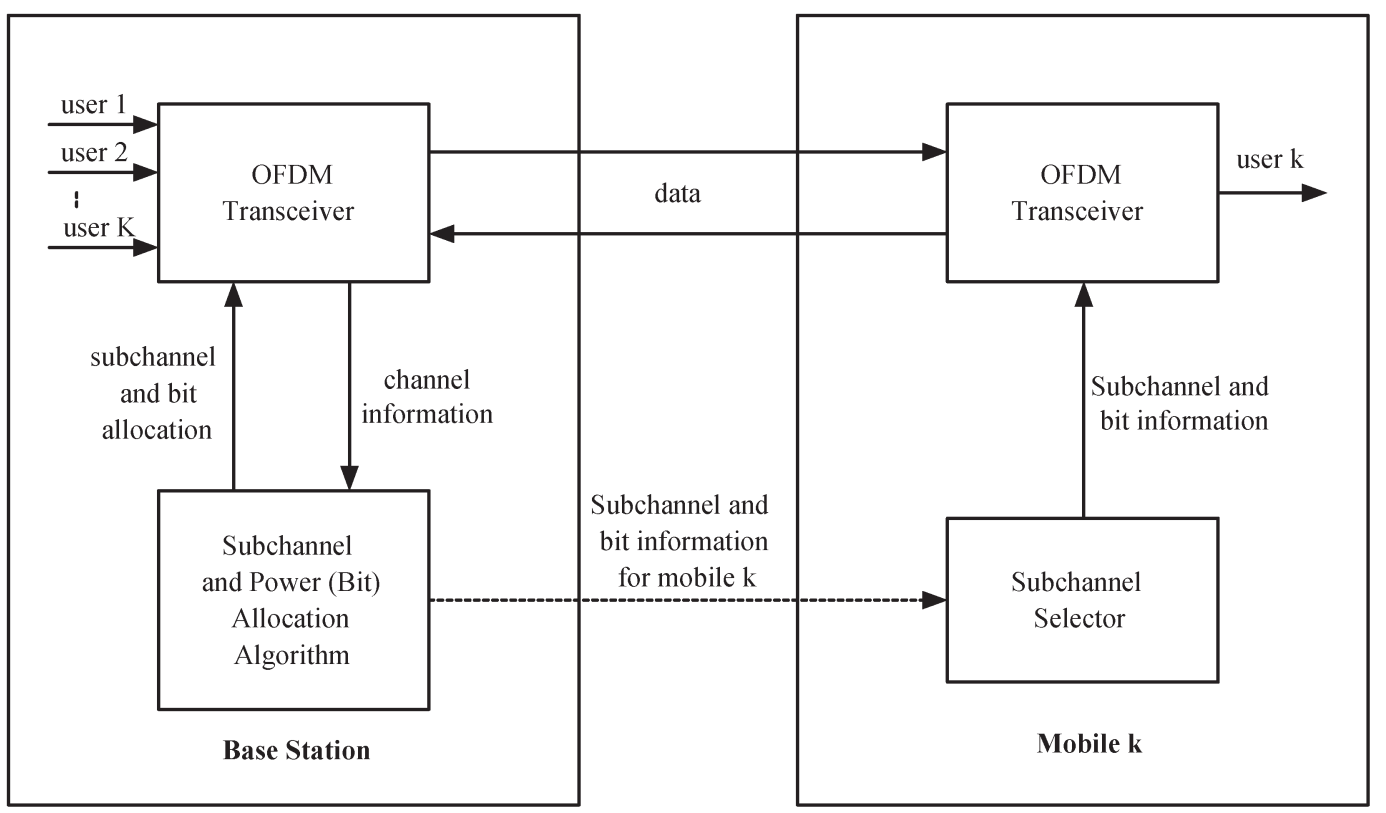

Fig. 1. Multiuser OFDM system block diagram.

out that in multiuser systems, channel fading can be exploited as a source of randomness, i.e., multiuser diversity. However, in some scenarios, due to the limited scatters in the environment and slow channel variation, the dynamic range of channel fluctuation in the time scale of interest may be small.

In this paper, we formulate a new optimization problem that balances the tradeoff between capacity and fairness. The objective function is still the sum capacity, but proportional fairness is assured by imposing a set of nonlinear constraints into the optimization problem. The definition of fairness is borrowed from the networking literature. In contrast to [9], where large channel fluctuations are intentionally created with "dumb" antennas for long-term proportional fairness resource allocation, this paper proposes an algorithm to maintain proportional rates among users for each channel realization, which ensures the rates of different users to be proportional in any time scale of interest. By formulating the problem this way, it will be shown that a high capacity for all users (even those with poor channel gains) can be achieved with low computational complexity.

This paper is organized as follows. Section II introduces the multiuser OFDM system model and presents the optimization objective function. In Section III, the optimal multiuser subchannel and power allocation is developed, and two approaches are discussed. In Section IV, the suboptimal algorithm is proposed, where subchannel and power allocations are carried out sequentially. Simulation results are presented in Section V and conclusions are drawn in Section VI. The Matlab simulation codes are available at $\langle$ http://www.ece.utexas.edu/ bevans/ projects/ofdm/software $\rangle$.

\section{SySTEM MOdEL}

A multiuser OFDM system is shown in Fig. 1. In the base station, all channel information is sent to the subchannel and power allocation algorithm through feedback channels from all mobile users. The resource allocation scheme made by the algorithm is forwarded to the OFDM transmitter. The transmitter then selects different numbers of bits from different users to form an OFDM symbol. The resource allocation scheme is updated as fast as the channel information is collected. In this paper, perfect instantaneous channel information is assumed to be available at the base station, and only the broadcast scenario is studied. It is also assumed that the subchannel and bit allocation information is sent to each user by a separate channel.

Throughout this paper, we assume a total of $K$ users in the system sharing $N$ subchannels, with total transmit power constraint $P_{\text {total }}$. Our objective is to optimize the subchannel and power allocation in order to achieve the highest sum errorfree capacity under the total power constraint. We use the equally weighted sum capacity as the objective function, but we introduce the idea of proportional fairness into the system by adding a set of nonlinear constraints. The benefit of introducing proportional fairness into the system is that we can explicitly control the capacity ratios among users, and generally ensure that each user is able to meet his target data rate, given sufficient total available transmit power.

Mathematically, the optimization problem considered in this paper is formulated as

$$
\begin{aligned}
& \max _{p_{k, n}, \rho_{k, n}} \sum_{k=1}^{K} \sum_{n=1}^{N} \frac{\rho_{k, n}}{N} \log _{2}\left(1+\frac{p_{k, n} h_{k, n}^{2}}{N_{0} \frac{B}{N}}\right) \\
& \text { subject to } \sum_{k=1}^{K} \sum_{n=1}^{N} p_{k, n} \leq P_{\text {total }} \\
& p_{k, n} \geq 0 \text { for all } k, n \\
& \rho_{k, n}=\{0,1\} \text { for all } k, n \\
& \\
& \sum_{k=1}^{K} \rho_{k, n}=1 \text { for all } n \\
& R_{1}: R_{2}: \ldots: R_{K}=\gamma_{1}: \gamma_{2}: \ldots: \gamma_{K}
\end{aligned}
$$


where $K$ is the total number of users, $N$ is the total number of subchannels, $N_{0}$ is the power spectral density of AWGN, $B$ and $P_{\text {total }}$ are the total available bandwidth and power, respectively, $p_{k, n}$ is the power allocated for user $k$ in the subchannel $n, h_{k, n}$ is the channel gain for user $k$ in subchannel $n$, and $\rho_{k, n}$ can only be either 1 or 0 , indicating whether subchannel $n$ is used by user $k$ or not. The fourth constraint shows that each subchannel can only be used by one user. The capacity for user $k$, denoted as $R_{k}$, is defined as

$$
R_{k}=\sum_{n=1}^{N} \frac{\rho_{k, n}}{N} \log _{2}\left(1+\frac{p_{k, n} h_{k, n}^{2}}{N_{0} \frac{B}{N}}\right) .
$$

Finally, $\left\{\gamma_{i}\right\}_{i=1}^{K}$ is a set of predetermined values that are used to ensure proportional fairness among users.

The fairness index is defined as

$$
\mathcal{F}=\frac{\left(\sum_{k=1}^{K} \gamma_{k}\right)^{2}}{K \sum_{k=1}^{K} \gamma_{k}^{2}}
$$

with the maximum value of 1 to be the greatest fairness case in which all users would achieve the same data rate. When all $\gamma_{i}$ terms are equal, the objective function in (1) is similar to the objective function of the max-min problem [7], since maximizing the sum capacity while making all $R_{k}$ terms equal is equivalent to maximizing the worst user's capacity. Hence, [7] is a special case of the proposed constrained-fairness problem.

\section{Optimal Subchannel Allocation AND POWER DISTRIBUTION}

The optimization problem in (1) is generally very hard to solve. It involves both continuous variables $p_{k, n}$ and binary variables $\rho_{k, n}$. Such an optimization problem is called a mixed binary integer programming problem. Furthermore, the nonlinear constraints in (1) increase the difficulty in finding the optimal solution, because the feasible set is not convex.

In a system with $K$ users and $N$ subchannels, there are $K^{N}$ possible subchannel allocations, since it is assumed that no subchannel can be used by more than one user. For a certain subchannel allocation, an optimal power distribution can be used to maximize the sum capacity, while maintaining proportional fairness. The optimal power distribution method is derived in the next section. The maximum capacity over all $K^{N}$ subchannel allocation schemes is the global maximum, and the corresponding subchannel allocation and power distribution is the optimal resource allocation scheme. However, it is prohibitive to find the global optimizer in terms of computational complexity. A suboptimal algorithm is derived in this paper to reduce the complexity significantly while still delivering performance close to the global optimum.

An alternative approach [5]-[7] to make the optimization problem in (1) easier to solve is to relax the constraint that subchannels can only be used by one user. Thus, $\rho_{k, n}$ is reinterpreted as the sharing factor of user $k$ to subchannel $n$, which can be any value on the half-open interval of $(0,1]$. The optimization in (1) can be transformed into

$$
\begin{aligned}
\min _{p_{k, n}, \rho_{k, n}}- & \sum_{k=1}^{K} \sum_{n=1}^{N} \frac{\rho_{k, n}}{N} \log _{2}\left(1+\frac{p_{k, n} h_{k, n}^{2}}{\rho_{k, n} N_{0} \frac{B}{N}}\right) \\
\text { subject to } & \sum_{k=1}^{K} \sum_{n=1}^{N} p_{k, n} \leq P_{\text {total }} \\
& p_{k, n} \geq 0 \text { for all } k, n \\
& \rho_{k, n} \in(0,1] \text { for all } k, n \\
K & \sum_{k=1}^{K} \rho_{k, n}=1 \text { for all } n \\
& R_{1}: R_{2}: \ldots: R_{K}=\gamma_{1}: \gamma_{2}: \ldots: \gamma_{K} .
\end{aligned}
$$

That is, the original maximization problem is transformed into a minimization problem. In the third constraint in (4), $\rho_{k, n}$ is not allowed to be zero, since the objective function is not defined for $\rho_{k, n}=0$. However, when $\rho_{k, n}$ is arbitrarily close to $0,\left(\rho_{k, n} / N\right) \log _{2}\left(1+\left(p_{k, n} h_{k, n}^{2}\right) /\left(\rho_{k, n} N_{0}(B / N)\right)\right)$ also approaches 0 . Thus, the nature of the objective function remains unchanged by excluding the case $\rho_{k, n}=0$.

A desirable property of the objective function in (4) is that it is convex on the set defined by the first two constraints. The convexity is shown in Appendix I. However, the nonlinear equality constraints make the feasible set nonconvex. In general, such optimization problems require linearization of the nonlinear constraints. The linearization procedure may lead the solution slightly off the feasible set defined by the nonlinear constraints. There is always a tradeoff between satisfaction of the constraints and improvement of the objective. Furthermore, it is still computationally complex to find the optimal solution. For these reasons, we propose a suboptimal technique in the next section.

\section{Suboptimal Subchannel Allocation AND POWER DISTRIBUTION}

Ideally, subchannels and power should be allocated jointly to achieve the optimal solution in (1). However, this poses a prohibitive computational burden at the base station in order to reach the optimal allocation. Furthermore, the base station has to rapidly compute the optimal subchannel and power allocation as the wireless channel changes. Hence, low-complexity suboptimal algorithms are preferred for cost-effective and delay-sensitive implementations. Separating the subchannel and power allocation is a way to reduce the complexity, because the number of variables in the objective function is almost reduced by half. Section IV-A discusses a subchannel allocation scheme. Section IV-B presents the optimal power distribution given a certain subchannel allocation. 


\section{A. Suboptimal Subchannel Allocation}

In this section, we discuss a suboptimal subchannel algorithm based on [7]. In the suboptimal subchannel allocation algorithm, equal power distribution is assumed across all subchannels. We define $H_{k, n}=\left(h_{k, n}^{2} / N_{0}(B / N)\right)$ as the channelto-noise ratio for user $k$ in subchannel $n$ and $\Omega_{k}$ is the set of subchannels assigned to user $k$. The algorithm can be described as

1) Initialization

a) Set $R_{k}=0, \Omega_{k}=\varnothing$ for $k=1,2, \ldots, K$ and $A=$ $\{1,2, \ldots, N\}$.

2) For $k=1$ to $K$,

a) find $n$ satisfying $\left|H_{k, n}\right| \geq\left|H_{k, j}\right|$ for all $j \in A$;

b) let $\Omega_{k}=\Omega_{k} \cup\{n\}, A=A-\{n\}$ and update $R_{k}$ according to (2).

3) While $A \neq \varnothing$,

a) find $k$ satisfying $R_{k} / \gamma_{k} \leq R_{i} / \gamma_{i}$ for all $i, 1 \leq i \leq K$;

b) for the found $k$, find $n$ satisfying $\left|H_{k, n}\right| \geq\left|H_{k, j}\right|$ for all $j \in A$;

c) for the found $k$ and $n$, let $\Omega_{k}=\Omega_{k} \cup\{n\}, A=A-$ $\{n\}$ and update $R_{k}$ according to (2)

The principle of the suboptimal subchannel algorithm is for each user to use the subchannels with high channel-to-noise ratio as much as possible. At each iteration, the user with the lowest proportional capacity has the option to pick which subchannel to use. The subchannel allocation algorithm is suboptimal, because equal power distribution in all subchannels is assumed. After subchannel allocation, only coarse proportional fairness is achieved. The goal of maximizing the sum capacity while maintaining proportional fairness is achieved by the power allocation in the next section.

\section{B. Optimal Power Distribution for a Fixed Subchannel Allocation}

To a certain determined subchannel allocation, the optimization problem is formulated as

$$
\begin{aligned}
\max _{p_{k, n}} & \sum_{k=1}^{K} \sum_{n \in \Omega_{k}} \frac{1}{N} \log _{2}\left(1+\frac{p_{k, n} h_{k, n}^{2}}{N_{0} \frac{B}{N}}\right) \\
\text { subject to } & \sum_{k=1}^{K} \sum_{n \in \Omega_{k}} p_{k, n} \leq P_{\text {total }} \\
& p_{k, n} \geq 0 \text { for all } k, n \\
& \Omega_{k} \text { are disjoint for all } k \\
& \Omega_{1} \cup \Omega_{2} \cup \ldots \cup \Omega_{K} \subseteq\{1,2, \ldots, N\} \\
& R_{1}: R_{2}: \ldots: R_{K}=\gamma_{1}: \gamma_{2}: \ldots: \gamma_{K}
\end{aligned}
$$

where $\Omega_{k}$ is the set of subchannels for user $k$, and $\Omega_{k}$ and $\Omega_{l}$ are mutually exclusive when $k \neq l$.
The optimization problem in (5) is equivalent to finding the maximum of the following cost function

$$
\begin{aligned}
L= & \sum_{k=1}^{K} \sum_{n \in \Omega_{k}} \frac{1}{N} \log _{2}\left(1+p_{k, n} H_{k, n}\right) \\
& +\lambda_{1}\left(\sum_{k=1}^{K} \sum_{n \in \Omega_{k}} p_{k, n}-P_{\text {total }}\right) \\
& +\sum_{k=2}^{K} \lambda_{k}\left(\sum_{n \in \Omega_{1}} \frac{1}{N} \log _{2}\left(1+p_{1, n} H_{1, n}\right)\right. \\
& \left.\quad-\frac{\gamma_{1}}{\gamma_{k}} \sum_{n \in \Omega_{k}} \frac{1}{N} \log _{2}\left(1+p_{k, n} H_{k, n}\right)\right)
\end{aligned}
$$

where $\left\{\lambda_{i}\right\}_{i=1}^{K}$ are the Lagrangian multipliers. We differentiate (6) with respect to $p_{k, n}$ and set each derivative to 0 to obtain

$$
\begin{aligned}
\frac{\partial L}{\partial p_{1, n}}= & \frac{1}{N \ln 2} \frac{H_{1, n}}{1+H_{1, n} p_{1, n}} \\
& +\lambda_{1}+\sum_{k=2}^{K} \lambda_{k} \frac{1}{N \ln 2} \frac{H_{1, n}}{1+H_{1, n} p_{1, n}}=0 \\
\frac{\partial L}{\partial p_{k, n}}= & \frac{1}{N \ln 2} \frac{H_{k, n}}{1+H_{k, n} p_{k, n}} \\
& +\lambda_{1}-\lambda_{k} \frac{\gamma_{1}}{\gamma_{k}} \frac{1}{N \ln 2} \frac{H_{k, n}}{1+H_{k, n} p_{k, n}}=0
\end{aligned}
$$

for $k=2,3, \ldots, K$ and $n \in \Omega_{k}$.

1) Power Distribution for a Single User: In this section, the optimal power distribution strategy for a single user $k$ is derived.

From either (7) or (8), we may obtain

$$
\frac{H_{k, m}}{1+H_{k, m} p_{k, m}}=\frac{H_{k, n}}{1+H_{k, n} p_{k, n}}
$$

for $m, n \in \Omega_{k}$ and $k=1,2, \ldots, K$. Without loss of generality, we assume that $H_{k, 1} \leq H_{k, 2} \leq \cdots \leq H_{k, N_{k}}$ for $k=$ $1,2, \ldots, K$ and $N_{k}$ is number of subchannels in $\Omega_{k}$. Thus, (9) can be rewritten as

$$
p_{k, n}=p_{k, 1}+\frac{H_{k, n}-H_{k, 1}}{H_{k, n} H_{k, 1}}
$$

for $n=1,2, \ldots, N_{k}$ and $k=1,2, \ldots, K$. Equation (10) shows that the power distribution for a single user $k$ on subchannel $n$. More power will be put into the subchannels with higher channel-to-noise ratio. This is the water-filling algorithm [13] in frequency domain.

By defining $P_{k, \text { tot }}$ as the total power allocated for user $k$ and using (10), $P_{k \text {,tot }}$ can be expressed as

$$
P_{k, \text { tot }}=\sum_{n=1}^{N_{k}} p_{k, n}=N_{k} p_{k, 1}+\sum_{n=2}^{N_{k}} \frac{H_{k, n}-H_{k, 1}}{H_{k, n} H_{k, 1}}
$$

for $k=1,2, \ldots, K$. 
2) Power Distribution Among Users: Once the set $\left\{P_{k, \text { tot }}\right\}_{k=1}^{K}$ is known, power allocation can be determined by (10) and (11). The total power constraint and capacity ratio constraints in (5) are used to obtain $\left\{P_{k, \text { tot }}\right\}_{k=1}^{K}$. With (9) and (11), the capacity ratio constraints can be expressed as

$$
\begin{aligned}
\frac{1}{\gamma_{1}} \frac{N_{1}}{N} & \left(\log _{2}\left(1+H_{1,1} \frac{P_{1, \text { tot }}-V_{1}}{N_{1}}\right)+\log _{2} W_{1}\right) \\
= & \frac{1}{\gamma_{k}} \frac{N_{k}}{N}\left(\log _{2}\left(1+H_{k, 1} \frac{P_{k, \text { tot }}-V_{k}}{N_{k}}\right)+\log _{2} W_{k}\right)
\end{aligned}
$$

for $k=2,3, \ldots, K$, where $V_{k}$ and $W_{k}$ are defined as

$$
V_{k}=\sum_{n=2}^{N_{k}} \frac{H_{k, n}-H_{k, 1}}{H_{k, n} H_{k, 1}}
$$

and

$$
W_{k}=\left(\prod_{n=2}^{N_{k}} \frac{H_{k, n}}{H_{k, 1}}\right)^{\frac{1}{N_{k}}}
$$

for $k=1,2, \ldots, K$.

Adding the total power constraints

$$
\sum_{k=1}^{K} P_{k, \text { tot }}=P_{\text {total }}
$$

there are $K$ variables $\left\{P_{k, \text { tot }}\right\}_{k=1}^{K}$ in the set of $K$ equations in (12) and (15). Solving the set of functions provides the optimal power allocation scheme. The equations are, in general, nonlinear. Iterative methods, such as the Newton-Raphson or quasi-Newton methods [15], can be used to obtain the solution, with a certain amount of computational effort. In the Newton-Raphson method, the computational complexity primarily comes from finding the update direction. In Appendix II, the computational complexity of each iteration is shown to be $\mathcal{O}(K)$. Under certain conditions, the optimal or near-optimal solution to the set of nonlinear equations can be found in one iteration. Two special cases are analyzed as follows.

1) Linear case: If $N_{1}: N_{2}: \ldots: N_{K}=\gamma_{1}: \gamma_{2}: \ldots: \gamma_{K}$, then the set of equations, i.e., (12) and (15), can be transformed into a set of linear equations by

$$
\left[\begin{array}{cccc}
1 & 1 & \ldots & 1 \\
1 & a_{2,2} & \ldots & 0 \\
\vdots & \vdots & \ddots & \vdots \\
1 & 0 & \ldots & a_{K, K}
\end{array}\right]\left[\begin{array}{c}
P_{1, \text { tot }} \\
P_{2, \text { tot }} \\
\vdots \\
P_{K, \text { tot }}
\end{array}\right]=\left[\begin{array}{c}
P_{\text {total }} \\
b_{2} \\
\vdots \\
b_{K}
\end{array}\right]
$$

where

$$
\begin{gathered}
a_{k, k}=-\frac{N_{1}}{N_{k}} \frac{H_{k, 1} W_{k}}{H_{1,1} W_{1}} \\
b_{k}=\frac{N_{1}}{H_{1,1} W_{1}}\left(W_{k}-W_{1}+\frac{H_{1,1} V_{1} W_{1}}{N 1}\right. \\
\left.\qquad \quad-\frac{H_{k, 1} V_{k} W_{k}}{N_{k}}\right)
\end{gathered}
$$

for $k=2,3, \ldots, K$. The matrix of $\left\{a_{i, i}\right\}_{i=2}^{K}$ in (16) has nonzero elements only on the first row, the first column, and the main diagonal. By substitution, the solution to (16) can be obtained with a computational complexity of $\mathcal{O}(K)$.

2) High channel-to-noise ratio case: In adaptive modulation, the linear condition rarely happens and the set of equations remains nonlinear, which requires considerably more computation to solve. However, if the channelto-noise ratio is high, approximations can be made to simplify the problem.

First, consider (13), in which $V_{k}$ could be relatively small compared to $P_{k \text {, tot }}$ if the channel-to-noise ratios are high. Furthermore, if adaptive subchannel allocation is used, the best subchannels will be chosen, and they have relatively small channel gain differences among them. Thus, the first approximation is $V_{k}=0$.

Second, assuming that the base station could provide a large amount of power and the channel-to-noise ratio is high, the term $H_{k, 1} P_{k \text {,tot }} / N_{k}$ is much larger than 1 .

With the above two approximations, (12) can be rearranged and simplified to be

$$
\left(\frac{H_{1,1} W_{1}}{N_{1}}\right)^{\frac{N_{1}}{\gamma_{1}}}\left(P_{1, \text { tot }}\right)^{\frac{N_{1}}{\gamma_{1}}}=\left(\frac{H_{k, 1} W_{k}}{N_{k}}\right)^{\frac{N_{k}}{\gamma_{k}}}\left(P_{k, \text { tot }}\right)^{\frac{N_{k}}{\gamma_{k}}}
$$

where $k=2,3, \ldots, K$.

Substituting (19) into (15), a single equation with the variable $P_{1 \text {,tot }}$ can be derived as

$$
\sum_{k=1}^{K} c_{k}\left(P_{1, \text { tot }}\right)^{d_{k}}-P_{\text {total }}=0
$$

where

$$
c_{k}= \begin{cases}1, & \text { if } k=1 \\ \frac{\left(\frac{H_{1,1} W_{1}}{N_{1}}\right)^{\frac{N_{1} \gamma_{k}}{N_{k} \gamma_{1}}}}{\frac{H_{k, 1} W_{k}}{N_{k}}}, & \text { if } k=2,3, \ldots, K\end{cases}
$$

and

$$
d_{k}= \begin{cases}1, & \text { if } k=1 \\ \frac{N_{1} \gamma_{k}}{N_{k} \gamma_{1}}, & \text { if } k=2,3, \ldots, K\end{cases}
$$

Numerical algorithms, such as Newton's root-finding method [14] or the false position method [14], can be applied to find the zero of (20).

\section{Existence of Power Allocation Scheme}

1) Solution to Single-User Power Allocation: For a certain user $k$, there is no power allocation if $V_{k}>P_{k \text {,tot }}$. This situation could happen when a subchannel is allocated to a user who does not have a high channel gain in that subchannel. The greedy water-filling algorithm would rather stop using this subchannel. In case this situation happens, the set of $\Omega_{k}$, as well as the corresponding values of $N_{k}, V_{k}$, and $W_{k}$, needs to be updated and the power allocation algorithm presented in this paper should again be executed, as shown in Fig. 2. 


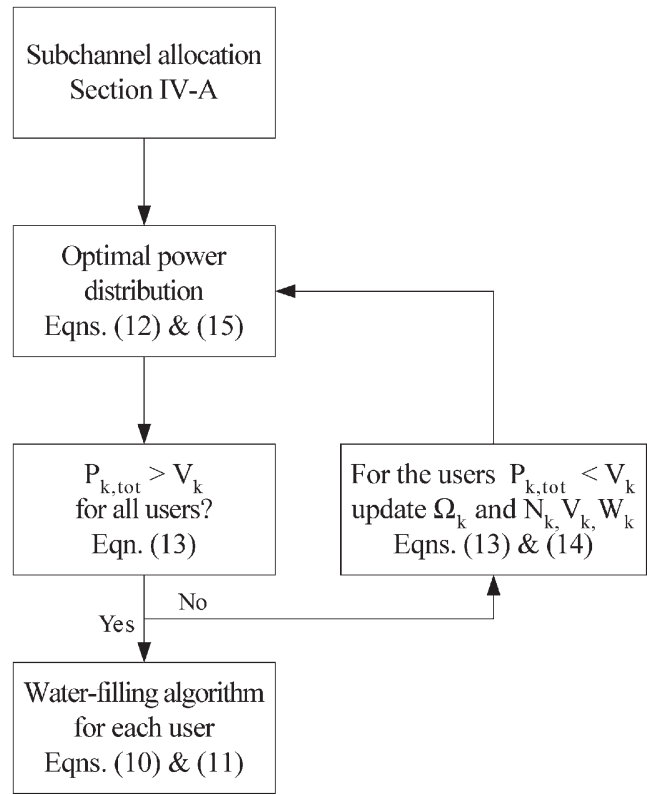

Fig. 2. Proposed resource allocation algorithm.

2) Solution to Multiuser Power Allocation: In case that the channel-to-noise ratio is high, there is one and only one solution to (20), since every item in the summation monotonically increases and (20) achieves different signs at $P_{1, \text { tot }}=0$ and $P_{1, \text { tot }}=P_{\text {total }}$. A numerical algorithm can be used to find the solution to (20). The complexity of finding the solution will primarily rely on the choice of the numerical algorithm and the precision required in the results. After $P_{1, \text { tot }}$ is found, $\left\{P_{k, \text { tot }}\right\}_{k=2}^{K}$ can be calculated using (19). Then the overall power allocation scheme can be determined by (10) and (11).

In general, it can be proven that there must be an optimal subchannel and power allocation scheme that satisfies the proportional fairness constraints and the total power constraint. Furthermore, the optimal scheme must utilize all available power. Several facts lead to the above conclusion. First, to a certain user, the capacity of the user is maximized if the waterfilling algorithm is adopted. Furthermore, the capacity function is continuous with respect to the total available power to that user. In other words, $R_{k}\left(P_{k, \text { tot }}\right)$ is continuous with $P_{k \text {,tot }}$. Second, if the optimal allocation scheme does not use all available transmit power, there is always a way to redistribute the unused power among users while maintaining the capacity ratio constraints, since $R_{k}\left(P_{k, \text { tot }}\right)$ is continuous with $P_{k \text {,tot }}$ for all $k$. Thus, the sum capacity is further increased. In Appendix II, we describe the Newton-Raphson method to find $P_{k \text {,tot }}$, without considering the constraints on $P_{k \text {,tot }}$, i.e., $P_{k \text {,tot }}>V_{k}$ for $k=1,2, \ldots, K$. If the Newton-Raphson method returns a nonfeasible $P_{k, \text { tot }}$, the set $\Omega_{k}$ and the associated $N_{k}, V_{k}$, and $W_{k}$ would need to be updated. The Newton-Raphson method should be performed until all $P_{k, \text { tot }}>V_{k}$.

\section{Complexity Analysis}

The best subchannel allocation scheme can be found by exhaustive search; i.e., for each subchannel allocation, one would

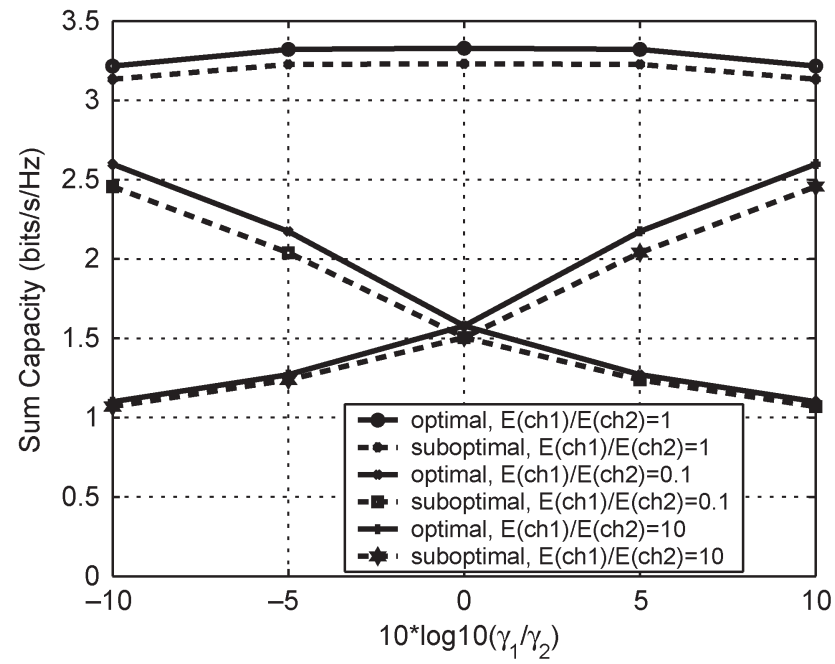

Fig. 3. Performance comparison of optimal and suboptimal algorithms, with two users and ten subchannels. AWGN power spectral density is $-70 \mathrm{~dB} \cdot \mathrm{W} / \mathrm{Hz}$. Total available bandwidth and transmit power are $1 \mathrm{MHz}$ and $1 \mathrm{~W}$, respectively.

run the optimal power allocation algorithm in Fig. 2, which has the computational complexity of $\mathcal{O}(K)$. The subcarrrier allocation that gives the highest sum capacity is the optimum. In a $K$-user $N$-subchannel system, it is prohibitive to find the global optimum, since there are $K^{N}$ possible subchannel allocations. The complexity of the proposed algorithm consists of two parts, namely 1) subchannel allocation with the complexity of $\mathcal{O}(K N)$ and 2) power allocation of $\mathcal{O}(K)$. Hence, the complexity of the proposed method is approximately on the order of $K^{N}$ times less than that of the optimal, because the power allocation is only executed once. The proposed method is described by Fig. 2 .

\section{NUMERICAL RESUlts}

In this section, we present simulation results to show the performance of the proposed resource allocation algorithm. We also show the tradeoff between sum capacity and the fairness constraints.

In all simulations presented in this section, the wireless channel is modeled as a frequency-selective channel consisting of six independent Rayleigh multipaths. Each multipath component is modeled by Clarke's flat fading model [12]. It is assumed that the power delay profile is exponentially decaying with $\mathrm{e}^{-2 l}$, where $l$ is the multipath index. Hence, the relative power of the six multipath components are $[0,-8.69,-17.37$, $-26.06,-34.74,-43.43] \mathrm{dB}$. The total available bandwidth and transmit power are $1 \mathrm{MHz}$ and $1 \mathrm{~W}$, respectively.

\section{A. System With Two Users and Ten Subchannels}

Fig. 3 shows the sum capacity versus $\gamma_{1} / \gamma_{2}$, from which the fairness index as defined in (3) can be calculated. Both the suboptimal results and the optimal results are plotted. A small number of users and subchannels are used in order to reduce the time to find the optimal solution. The sum capacities shown in Fig. 3 are averaged over 200 channel realizations. In Fig. 3, we 
can see that the sum capacity is not very sensitive to the fairness constraint ratio $\gamma_{1} / \gamma_{2}$ when there is no path loss difference between the two users. However, when there exists path loss difference, e.g., $10 \mathrm{~dB}$, the sum capacity varies greatly with the fairness constraint ratio. For example, when the averaged channel power of user 1 , denoted as $E\left(\mathrm{ch}_{1}\right)$, is $10 \mathrm{~dB}$ higher than average channel power of user 2 , denoted as $E\left(\mathrm{ch}_{2}\right)$, the sum capacity reduces as $\gamma_{1} / \gamma_{2}$ decreases. The reason is that as $\gamma_{1} / \gamma_{2}$ decreases, more priority is assigned to user 2. Hence, user 2 will be assigned most of the available resources, i.e., power and bandwidth, which, consequently, lowers the sum capacity, since the average channel power of user 2 is $10 \mathrm{~dB}$ lower than user 1.

In Fig. 3, the proposed method achieves about $95 \%$ of the optimal performance in a two-user ten-subchannel system. Although in a real cellular or wireless local area network (LAN) system, the number of users and subchannels is much larger, we still expect the proposed method to perform close to the optimum, because the subchannel allocation algorithm is designed to utilize the subchannels with large channel-to-noise ratio as much as possible, and the power distribution is always optimal for any determined subchannel allocation.

\section{B. Comparison With the Method in [7]}

The objective in [7] is to maximize the minimum user's capacity. By setting $\gamma_{1}: \gamma_{2}: \ldots: \gamma_{K}=1: 1: \ldots: 1$, the objective of the optimization problem in (1) is identical to the one in [7], since the worst user's capacity is maximized when all users have the same capacity and the sum capacity is maximized. Hence, the problem in [7] is a special case of the framework presented in this paper. In this section of simulations, the worst user's capacity is compared. In [7], a suboptimal algorithm is proposed to achieve near-optimal capacity using adaptive subchannel allocation, but an equal power distribution is assumed. When the number of users increases, the equal power distribution does not equalize every user's capacity. By transferring power from the users with high capacity to the users with low capacity, the worst user's capacity could even be increased. For the purpose of comparison, we use the suboptimal algorithm in [7], which is a special case of the subchannel allocation algorithm in this paper, to allocate the subchannels first and then apply the optimal power allocation scheme proposed in this paper. Both of these adaptive schemes are compared with the fixed TDMA resource allocation scheme.

The wireless channel is modeled as before, and the total transmit power available at the base station is $1 \mathrm{~W}$. The power spectral density of $\mathrm{AWGN}$ is $-80 \mathrm{~dB} \cdot \mathrm{W} / \mathrm{Hz}$, and the total bandwidth is $1 \mathrm{MHz}$, which is divided into 64 subchannels. The maximum path loss difference is $40 \mathrm{~dB}$, and the user locations are assumed to be uniformly distributed. In this part of the simulation, the subchannel SNR is high, hence, the power allocation algorithm can be reduced to the high channel-tonoise case discussed in Section IV-B2.

Fig. 4 shows the capacity versus number of users in an OFDM system. In Fig. 4, it can be seen that adaptive resource allocation can achieve significant capacity gain over nonadap-

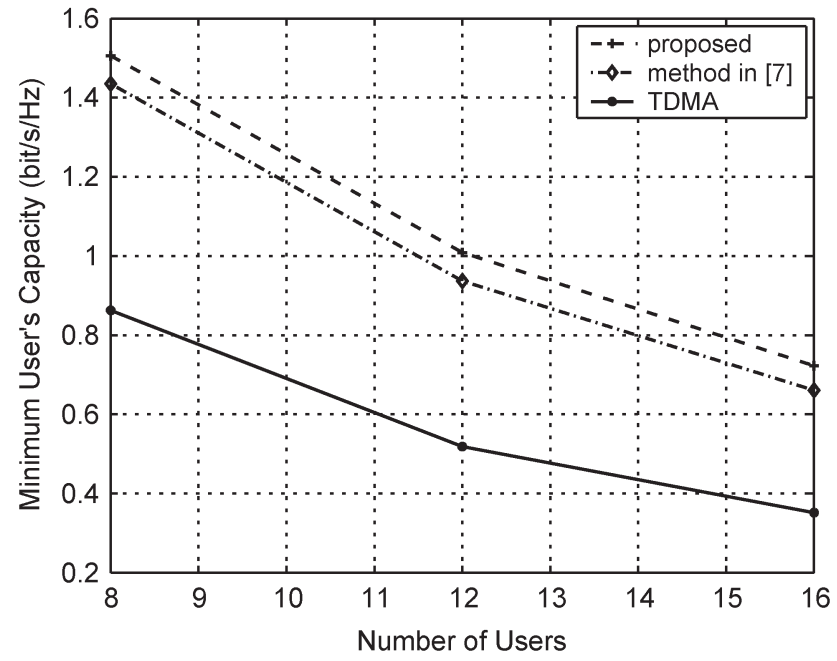

Fig. 4. Minimum user's capacity in multiuser OFDM versus number of users. The total power available at the base station is $1 \mathrm{~W}$. The power spectral density of AWGN is $-80 \mathrm{~dB} \cdot \mathrm{W} / \mathrm{Hz}$. The overall bandwidth is $1 \mathrm{MHz}$, which is divided into 64 subchannels. This plot shows the capacity gain of optimal power allocation over equal power distribution, as well as the capacity gain of adaptive resource allocations over a static TDMA system.

tive TDMA. In addition, the adaptive scheme with optimal power allocation achieves even higher capacity than the scheme with equal power distribution. Notice that this capacity gain is purely from the optimal power allocation algorithm, since both adaptive resource allocation algorithms adopt the same subchannel allocation. Fig. 4 also shows that the capacity gain over TDMA increases when the number of users increases. This can be explained by multiuser diversity: The more users in the system, the lower the probability that a given subchannel is in a deep fade for all users. In a system of 16 users, the adaptive scheme with the proposed optimal power allocation achieves $17 \%$ more capacity gain than the scheme with equal power distribution, when compared to fixed TDMA.

\section{Comparison With the Method in [6]}

In this subsection, we compare the sum capacity achieved by the proposed algorithm with the method in [6]. The simulation parameters are the same as the previous section, i.e., the total available bandwidth is $1 \mathrm{MHz}$, the total transmit power at the base station is $1 \mathrm{~W}$, the AWGN power density is $-80 \mathrm{~dB} \cdot \mathrm{W} / \mathrm{Hz}$, and the number of subchannels is $N=64$.

In Fig. 5, we show the sum capacity of our proposed resource allocation algorithm in an eight-user OFDM system versus different fairness constraints, which are defined in Table I. The average channel power of user 2 to user 8 is the same, while the average channel power of user 1 is $10 \mathrm{~dB}$ higher than the other seven users. In Fig. 5, we also show that

1) the sum capacity achieved by the method in [6];

2) the capacity achieved by a static TDMA system, in which each user is allocated an equal share of time slots and equal transmit power;

3) the capacities of two types of single-user systems, one for the user with high average channel power, and the other for those with low average channel power. 


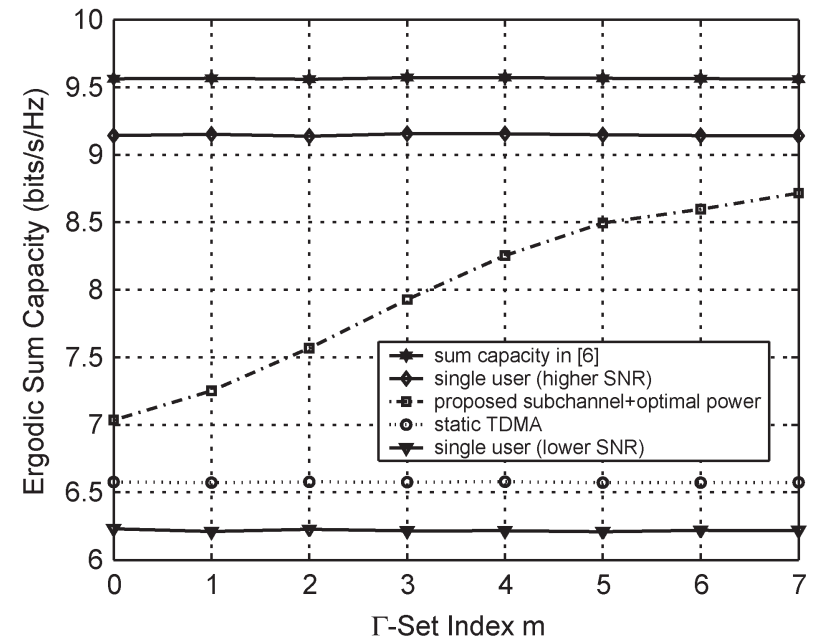

Fig. 5. Ergodic sum capacity of multiuser OFDM systems versus various $\Gamma$-sets. The total transmit power is $1 \mathrm{~W}$. The total bandwidth is $1 \mathrm{MHz}$, which is divided into 64 subchannels. The AWGN noise power density is $-80 \mathrm{~dB} \cdot \mathrm{W} / \mathrm{Hz}$. The number of users is 8 . The average channel power is $E\left(\operatorname{ch}_{1}\right)=10 E\left(\operatorname{ch}_{k}\right)$ for $k=2, \ldots, 8$. The rate constraints are $\gamma_{1}=2^{m}$ and $\gamma_{k}=1$ for $k \neq 1$, where $m$ is the $\Gamma$-set index.

All sum capacities shown in Fig. 5 are ergodic capacities averaged over $5 \times 10^{4}$ channel realizations. It can be seen that the sum capacity maximization method in [6] achieves the maximum sum capacity, because all resources are allocated to the users with the best channel gains. The capacity achieved by the proposed algorithm varies as the rate constraint changes. As more priority is allocated to user 1 , i.e., as the $\Gamma$-set index increases, higher sum capacity is achieved. This is reasonable, since user 1 has higher average channel gain and, hence, can more efficiently utilize the resources.

Fig. 6 shows the normalized ergodic capacity distribution among users for $\Gamma$-set index 3 in Table I, where $\gamma_{1}=8$ and $\gamma_{2}=\gamma_{2}=\cdots=\gamma_{8}=1$. With the proposed subchannel and power allocation algorithm, the capacity is distributed very well among users according to the rate constraints. However, for the capacity maximization method in [6], user 1 gets most of the resources and, hence, achieves a significant part of the sum capacity. Static TDMA tends to allocate similar capacity to each user, since all users get the same opportunity to transmit. Notice that the capacity distribution of the method in [6] and static TDMA cannot be changed by varying the $\Gamma$-set values, because there is no fairness control mechanism in these systems.

Since the problem formulation in (1) is to allocate resources to satisfy the rate constraints strictly for each channel realization, we define a quantity to measure how well the rate constraints are satisfied. Let $R_{k, i}$ be the capacity of user $k$ for a certain channel realization $i, \widetilde{R}_{k, i}=R_{k, i} / \sum_{k=1}^{K} R_{k, i}$ be the normalized capacity for user $k$, and $\widetilde{\gamma}_{k}=\gamma_{k} / \sum_{k=1}^{K} \gamma_{k}$ be the normalized rate constraint. The normalized rate constraint deviation measure for channel realization $i$ is defined as

$$
\mathcal{D}_{i}=\frac{\sum_{k=1}^{K}\left|\widetilde{R}_{k, i}-\widetilde{\gamma}_{k}\right|}{\max _{\widetilde{R}_{k, i}} \sum_{k=1}^{K}\left|\widetilde{R}_{k, i}-\widetilde{\gamma}_{k}\right|} .
$$

Notice that the denominator in (23) refers to the maximum deviation over all possible $\widetilde{R}_{k, i}$ values. It is shown in Appendix III that

$$
\max _{\widetilde{R}_{k, i}} \sum_{k=1}^{K}\left|\widetilde{R}_{k, i}-\widetilde{\gamma}_{k}\right|=2-2 \min _{k} \widetilde{\gamma}_{k} .
$$

Table I shows the averaged rate constraint deviations, denoted as $\overline{\mathcal{D}}=\sum_{i=1}^{I} \mathcal{D}_{i} / I$, where $I$ is the total number of channel realizations of the eight-user OFDM system. The rate constraint deviation of the proposed subchannel and power allocation is orders of magnitude smaller than those achieved by the method in [6] and the static TDMA. In other words, the price of maximizing ergodic capacity is that the short-term data rates vary widely and users may have poor performance over a certain block of time.

Fig. 7 shows the ergodic sum capacities in a multiuser OFDM systems with 16 users. The simulation parameters are the same as those in the previous eight-user system. The average channel power of the first four users are $10 \mathrm{~dB}$ higher than the rest of 12 users. The $\Gamma$-set index and the rate constraint deviations are shown in Table II. Fig. 8 shows the normalized average sum capacity distribution among users with $\gamma_{1}=\cdots=\gamma_{4}=8$ and $\gamma_{5}=\cdots=\gamma_{16}=1$. It should be noted that higher sum capacity is achieved by the method in [6] in this 16-user OFDM system, since more users with high channel power are in this system, hence, more multiuser diversity. However, it can be seen from Fig. 8 that the users with lower average channel power, i.e., users 5-16, get very small portions of the sum capacity, since in most channel realizations, the subchannels and power are allocated to the users with larger subchannel gains.

\section{CONCLUSION}

In this paper, we present a resource allocation framework in MU-OFDM systems to achieve variable proportional rate constraints. For different rate constraints, i.e., $\left\{\gamma_{k}\right\}_{k=1}^{K}$, different proportional rates can be achieved among users. The term "variable" refers to the facts that the rate constraints can be configured at the base station and, hence, rate allocation among users is flexible.

The proposed optimization problem considers maximizing the sum capacity while maintaining proportional fairness among users for each channel realization. The algorithm to find the optimal solution is discussed, and a low complexity suboptimal algorithm, which reduces complexity from $\mathcal{O}\left(K^{N}\right)$ to $\mathcal{O}(K N)$, is also proposed. In the suboptimal algorithm, subchannel and power allocation are carried out separately. The optimal power allocation to a determined subchannel scheme is developed. A two-step procedure may be taken to get the optimal power distribution. First, a set of nonlinear equations has to be solved in order to get the power distribution among users. Then to a particular user, the greedy water-filling algorithm is adopted to maximize the capacity. The existence of power allocation is also discussed.

Simulation results show that the suboptimal algorithm can achieve above $95 \%$ of the optimal performance in a 
TABLE I

Rate Constraints ( $\Gamma$-Sets) And Rate Constraint Deviations for Figs. 5 And $6, K=8$

\begin{tabular}{c|c|c|c|c|c|c|c|c}
\hline$\Gamma$-Set Index $m$ & 0 & 1 & 2 & 3 & 4 & 5 & 6 & 7 \\
\hline \hline$\gamma_{1}=2^{m}$ & $2^{0}$ & $2^{1}$ & $2^{2}$ & $2^{3}$ & $2^{4}$ & $2^{5}$ & $2^{6}$ & $2^{7}$ \\
\hline$\gamma_{2}=\gamma_{3}=\cdots=\gamma_{8}$ & 1 & 1 & 1 & 1 & 1 & 1 & 1 & 1 \\
\hline$\overline{\mathcal{D}}$, proposed subchannel + optimal power & 0.0026 & 0.0024 & 0.0020 & 0.0015 & 0.0012 & 0.0010 & 0.0013 & 0.0012 \\
\hline$\overline{\mathcal{D}}$, sum capacity maximization in [6] & 0.8848 & 0.7825 & 0.6441 & 0.5004 & 0.3878 & 0.3216 & 0.2902 & 0.2751 \\
\hline$\overline{\mathcal{D}}$, static TDMA & 0.1118 & 0.1114 & 0.2247 & 0.3867 & 0.5453 & 0.6633 & 0.7377 & 0.7799 \\
\hline
\end{tabular}

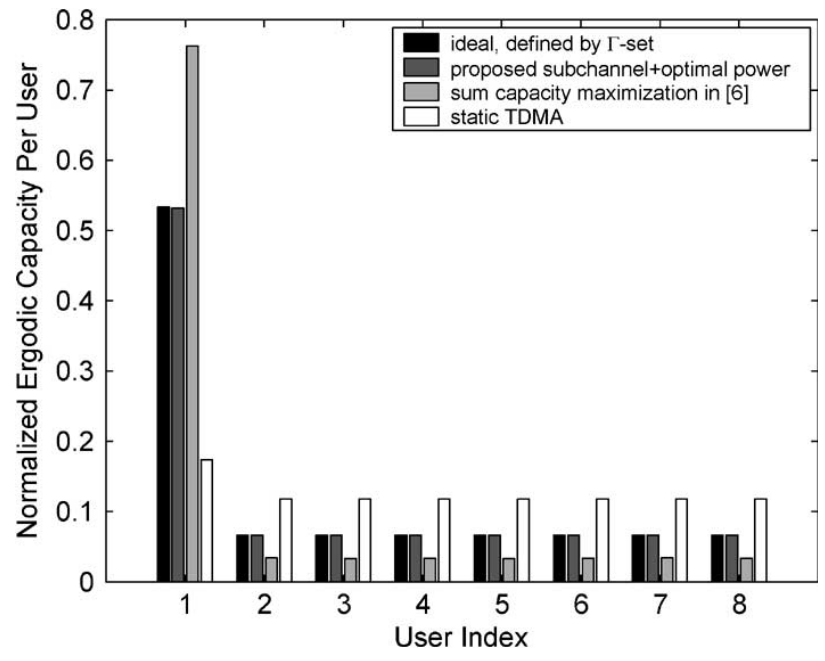

Fig. 6. Normalized ergodic sum capacity distribution among users. The simulation parameters are the same as in Fig. 5. The rate constraint is $\gamma_{1}=8$ and $\gamma_{2}=\cdots=\gamma_{8}=1$.

two-user system. It is also shown that in a system of 16 users, the proposed optimal power allocation achieves $17 \%$ more capacity over fixed TDMA than the max-min method in [7]. We also show that with the proposed resource allocation algorithm, the sum capacity is distributed more fairly among users than the sum capacity maximization algorithm in [6].

\section{APPENDIX I}

\section{CONVEXITY OF THE OBJECTIVE FUNCTION IN THE RELAXED OPTIMIZATION PROBLEM}

First, consider the following function

$$
f\left(\rho_{k, n}, p_{k, n}\right)=\rho_{k, n} \log _{2}\left(1+\frac{p_{k, n} H_{k, n}}{\rho_{k, n}}\right)
$$

where $H_{k, n}=h_{k, n}^{2} /\left(N_{0}(B / N)\right)$.

The Jacobian of $f\left(\rho_{k, n}, p_{k, n}\right)$ is calculated as

$$
\begin{aligned}
& \nabla f\left(\rho_{k, n}, p_{k, n}\right) \\
& \quad=\left[\begin{array}{l}
\log _{2}\left(1+\frac{p_{k, n} H_{k, n}}{\rho_{k, n}}\right)-\frac{1}{\ln 2} \frac{p_{k, n} H_{k, n}}{\rho_{k, n}+p_{k, n} H_{k, n}} \\
\frac{1}{\ln 2} \frac{\rho_{k, n} H_{k, n}}{\rho_{k, n}+p_{k, n} H_{k, n}}
\end{array}\right] .
\end{aligned}
$$

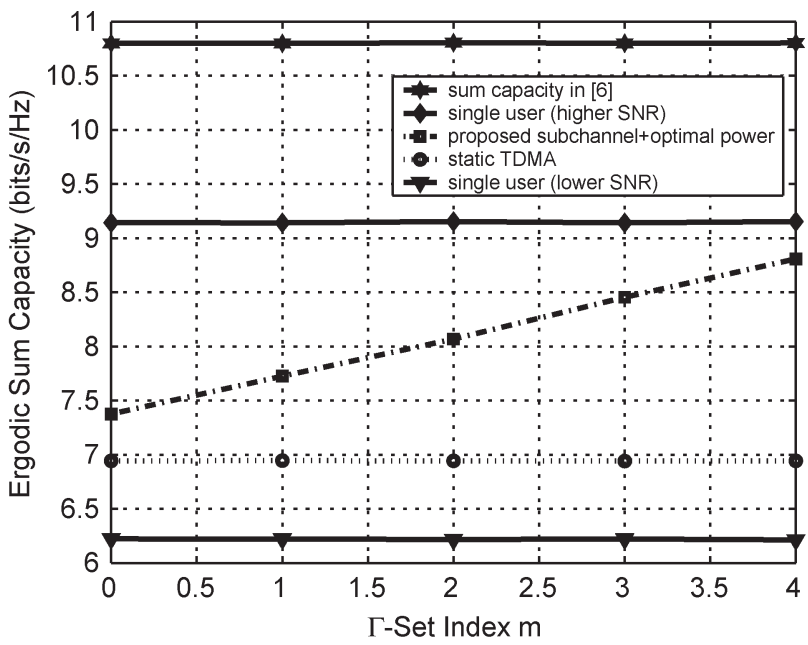

Fig. 7. Ergodic sum capacity of multiuser OFDM systems versus various $\Gamma$-sets. The total transmit power is $1 \mathrm{~W}$. The total bandwidth is $1 \mathrm{MHz}$, which is divided into 64 subchannels. The AWGN noise power density is $-80 \mathrm{~dB} \cdot \mathrm{W} / \mathrm{Hz}$. The number of users is 16 . The average channel power of users $1-4$ is $10 \mathrm{~dB}$ higher than the rest of 12 users. The rate constraints are $\gamma_{k}=2^{m}$ for $k=1, \ldots, 4$ and $\gamma_{k}=1$ for $k=5, \ldots, 16$, where $m$ is the $\Gamma$-set index.

The Hessian of $f\left(\rho_{k, n}, p_{k, n}\right)$ is calculated as

$$
\begin{aligned}
& \nabla^{2} f\left(\rho_{k, n}, p_{k, n}\right) \\
& \quad=\frac{1}{\ln 2} \frac{p_{k, n} H_{k, n}^{2}}{\left(\rho_{k, n}+p_{k, n} H_{k, n}\right)^{2}}\left[\begin{array}{cc}
-\frac{p_{k, n}}{\rho_{k, n}} & 1 \\
1 & -\frac{\rho_{k, n}}{p_{k, n}}
\end{array}\right] .
\end{aligned}
$$

Since $\rho_{k, n}, p_{k, n}$, and $H_{k, n}$ are all positive, it is not difficult to see that the Hessian of $f\left(\rho_{k, n}, p_{k, n}\right)$ is negative semidefinite and, hence, $f\left(\rho_{k, n}, p_{k, n}\right)$ is concave. Thus, the Hessian of $-f\left(\rho_{k, n}, p_{k, n}\right)$ is positive semidefinite and $-f\left(\rho_{k, n}, p_{k, n}\right)$ is convex. The objective function in (4) can be expressed as

$$
\sum_{k=1}^{K} \sum_{n=1}^{N} \frac{1}{N}\left(-f\left(\rho_{k, n}, p_{k, n}\right)\right)
$$

and, thus, is a summation of a set of convex functions, which is also convex.

\section{APPENDIX II}

\section{Newton-Raphson Method to Solve the NONLINEAR EQUATIONS}

In the following, we describe the major steps to find the power allocation with Newton-Raphson method. 
TABLE II

Rate Constraints ( $\Gamma$-Sets) and Rate Constraint Deviations for Figs. 7 And $8, K=16$

\begin{tabular}{c|c|c|c|c|c}
\hline$\Gamma$-Set Index $m$ & 0 & 1 & 2 & 3 & 4 \\
\hline \hline$\gamma_{1}=\gamma_{2}=\cdots=\gamma_{4}=2^{m}$ & $2^{0}$ & $2^{1}$ & $2^{2}$ & $2^{3}$ & $2^{4}$ \\
\hline$\gamma_{5}=\gamma_{6}=\cdots=\gamma_{16}$ & 1 & 1 & 1 & 1 & 1 \\
\hline$\overline{\mathcal{D}}$, proposed subchannel + optimal power & 0.0015 & 0.0015 & 0.0013 & 0.0012 & 0.0018 \\
\hline$\overline{\mathcal{D}}$, sum capacity maximization in [6] & 0.9238 & 0.8361 & 0.7438 & 0.6662 & 0.6133 \\
\hline$\overline{\mathcal{D}}$,static TDMA & 0.1150 & 0.1093 & 0.2548 & 0.4071 & 0.5193 \\
\hline
\end{tabular}

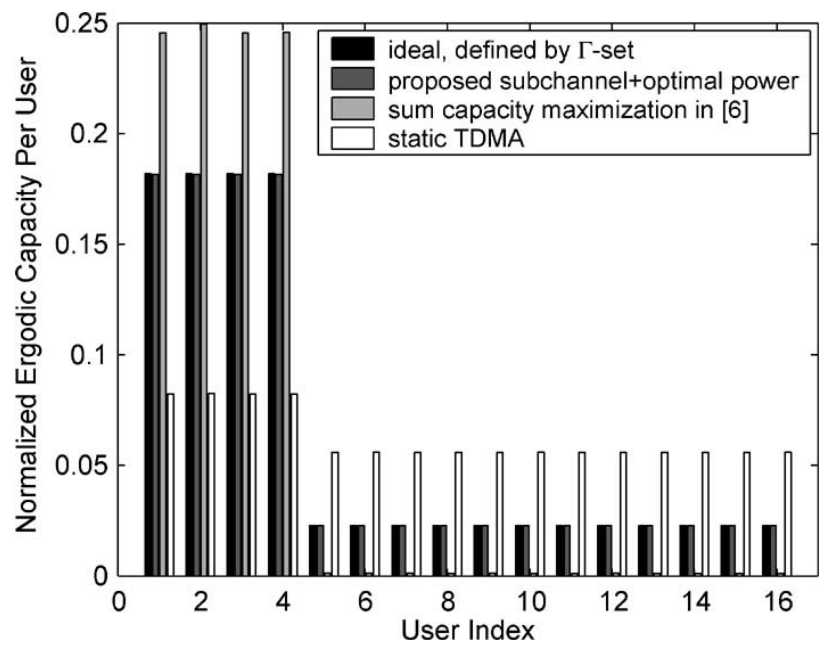

Fig. 8. Normalized ergodic sum capacity distribution among users. The simulation parameters are the same as in Fig. 7. The rate constraint is $\gamma_{1}=$ $\cdots=\gamma_{4}=8$ and $\gamma_{5}=\cdots=\gamma_{16}=1$.

Denote the variables as

$$
\mathbf{P}=\left[P_{1, \text { tot }} P_{2, \text { tot }} \ldots P_{K, \text { tot }}\right]^{+}
$$

where $[\bullet]^{+}$represents the operation of matrix transpose.

Furthermore, define a square system of equations $\mathbf{g}(\mathbf{P})=\mathbf{0}$ where

$$
g_{1}(\mathbf{P})=\sum_{k=1}^{K} P_{k, \text { tot }}-P_{\text {total }}=0
$$

and

$$
\begin{aligned}
g_{k}(\mathbf{P})= & \frac{N_{1}}{N}\left(\log _{2}\left(1+H_{1,1} \frac{P_{1, \text { tot }}-V_{1}}{N_{1}}\right)+\log _{2} W_{1}\right) \\
& -\frac{\gamma_{1}}{\gamma_{k}} \frac{N_{k}}{N}\left(\log _{2}\left(1+H_{k, 1} \frac{P_{k, \text { tot }}-V_{k}}{N_{k}}\right)+\log _{2} W_{k}\right) \\
= & 0
\end{aligned}
$$

for $k=2, \ldots, K$.

Denote $\triangle \mathbf{P}$ as the update direction. The major step in Newton-Raphson method is to solve the following equation to find $\triangle \mathbf{P}$

$$
\mathcal{J}(\mathbf{P}) \triangle \mathbf{P}=-\mathbf{g}(\mathbf{P})
$$

and update $\mathbf{P}$ as

$$
\mathbf{P}=\mathbf{P}+\triangle \mathbf{P}
$$

where

$$
\mathcal{J}(\mathbf{P})=\left[\begin{array}{cccc}
\frac{\partial g_{1}}{\partial P_{1, \text { tot }}} & \frac{\partial g_{1}}{\partial P_{2} \text {,tot }} & \cdots & \frac{\partial g_{1}}{\partial P_{K, \text { tot }}} \\
\frac{\partial g_{2}}{\partial P_{1, \text { tot }}} & \frac{\partial g_{2}}{\partial P_{2, \text { tot }}} & \cdots & \frac{\partial g_{2}}{\partial P_{K, \text { tot }}} \\
\vdots & \vdots & \ddots & \vdots \\
\frac{\partial g_{K}}{\partial P_{1, \text { tot }}} & \frac{\partial g_{K}}{\partial P_{2, \text { tot }}} & \cdots & \frac{\partial g_{K}}{\partial P_{K, \text { tot }}}
\end{array}\right]
$$

is the Jacobian matrix of $\mathbf{g}(\mathbf{P})$ evaluated at $\mathbf{P}$.

It is true that the computational complexity is still high, since, in general, a matrix inversion or LU decomposition has to be performed in order to get $\triangle \mathbf{P}$ each iteration. Fortunately, the Jacobian matrix of $\mathbf{g}(\mathbf{P})$ has a good structure that can be

$$
\begin{aligned}
& \mathcal{J}(\mathbf{P})=\left[\begin{array}{cccc}
\frac{\partial g_{1}}{\partial P_{1, \text { tot }}} & \frac{\partial g_{1}}{\partial P_{2, \text { tot }}} & \cdots & \frac{\partial g_{1}}{\partial P_{K, \text { tot }}} \\
\frac{\partial g_{2}}{\partial P_{1, \text { tot }}} & \frac{\partial g_{2}}{\partial P_{2, \text { tot }}} & \cdots & \frac{\partial g_{2}}{\partial P_{K, \text { tot }}} \\
\vdots & \vdots & \ddots & \vdots \\
\frac{\partial g_{K}}{\partial P_{1, \text { tot }}} & \frac{\partial g_{K}}{\partial P_{2, \text { tot }}} & \cdots & \frac{\partial g_{K}}{\partial P_{K, \text { tot }}}
\end{array}\right]
\end{aligned}
$$

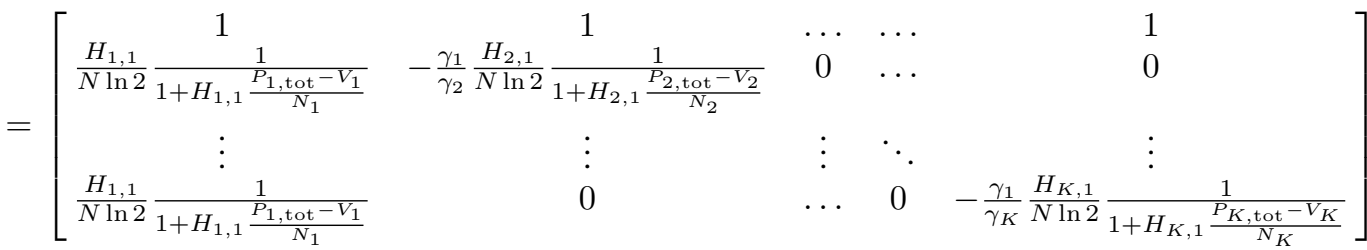


fully utilized to reduce the computational complexity [see (35), shown at the bottom of the previous page]. Every except the first row of the Jacobian matrix has only two nonzero entries. By substitution, $\triangle \mathbf{P}$ can be calculated with the complexity of $\mathcal{O}(K)$.

\section{APPENDIX III \\ Calculation of MaXimum Deviation DEFINED IN (23)}

Notice that $\sum_{k=1}^{K} \widetilde{R}_{k, i}=1$ and $\sum_{k=1}^{K} \widetilde{\gamma}_{k}=1$. Hence, there must exist some $k$, such that $\widetilde{R}_{k, i}-\widetilde{\gamma}_{k}<0$. Without loss of generality, assume that $\widetilde{R}_{k, i}-\widetilde{\gamma}_{k}<0$ for $k=1,2, \ldots, k^{*}$ and $\widetilde{R}_{k, i}-\widetilde{\gamma}_{k} \geq 0$ for $k=k^{*}+1, \ldots, K$, then the objective function can be written as

$$
\begin{aligned}
\sum_{k=1}^{K}\left|\widetilde{R}_{k, i}-\widetilde{\gamma}_{k}\right| & \\
= & \sum_{k=1}^{k^{*}}\left(\widetilde{\gamma}_{k}-\widetilde{R}_{k, i}\right)+\sum_{k=k^{*}+1}^{K}\left(\widetilde{R}_{k, i}-\widetilde{\gamma}_{k}\right) \\
= & \sum_{k=1}^{k^{*}} \widetilde{\gamma}_{k}-\sum_{k=k^{*}+1}^{K} \widetilde{\gamma}_{k}+\sum_{k=k^{*}+1}^{K} \widetilde{R}_{k, i}-\sum_{k=1}^{k^{*}} \widetilde{R}_{k, i} \\
= & \sum_{k=1}^{k^{*}} \widetilde{\gamma}_{k}+\sum_{k=k^{*}+1}^{K} \widetilde{\gamma}_{k}-2 \sum_{k=k^{*}+1}^{K} \widetilde{\gamma}_{k} \\
& +\sum_{k=k^{*}+1}^{K} \widetilde{R}_{k, i}+\sum_{k=1}^{k^{*}} \widetilde{R}_{k, i}-2 \sum_{k=1}^{k^{*}} \widetilde{R}_{k, i} \\
\leq & 1-2 \min _{k} \widetilde{\gamma}_{k}+1 \\
= & 2-2 \min _{k} \widetilde{\gamma}_{k} .
\end{aligned}
$$

Let $\arg \min _{k} \widetilde{\gamma}_{k}=k_{\text {min }}$, then one maximizer of the objective function in (24) is $\widetilde{R}_{k, i}=1$ for $k=k_{\min }$ and $\widetilde{R}_{k, i}=0$ otherwise.

\section{ACKNOWLEDGMENT}

The authors appreciate the useful comments from the anonymous reviewers.

\section{REFERENCES}

[1] H. Sampath, S. Talwar, J. Tellado, V. Erceg, and A. Paulraj, "A fourthgeneration MIMO-OFDM broadband wireless system: Design, performance, and field trial results," IEEE Commun. Mag., vol. 40, no. 9, pp. 143-149, Sep. 2002.

[2] T. S. Rappaport, A. Annamalai, R. M. Buehrer, and W. H. Tranter, "Wireless communications: Past events and a future perspective," IEEE Commun. Mag., vol. 40, no. 5, pp. 148-161, May 2002.

[3] J. A. C. Bingham, "Multicarrier modulation for data transmission: An idea whose time has come," IEEE Commun. Mag., vol. 28, no. 5, pp. 5-14, May 1990.

[4] E. Lawrey, "Multiuser OFDM," in Proc. Int. Symp. Signal Processing and Its Applications, Brisbane, Australia, 1999, pp. 761-764.

[5] C. Y. Wong, R. S. Cheng, K. B. Letaief, and R. D. Murch, "Multicarrier OFDM with adaptive subcarrier, bit, and power allocation," IEEE J. Sel. Areas Commun., vol. 17, no. 10, pp. 1747-1758, Oct. 1999.
[6] J. Jang and K. B. Lee, "Transmit power adaptation for multiuser OFDM systems," IEEE J. Sel. Areas Commun., vol. 21, no. 2, pp. 171-178, Feb. 2003.

[7] W. Rhee and J. M. Cioffi, "Increasing in capacity of multiuser OFDM system using dynamic subchannel allocation," in Proc. IEEE Int. Vehicular Tech. Conf., Tokyo, Japan, May 2000, vol. 2, pp. 1085-1089.

[8] I. Kim, H. L. Lee, B. Kim, and Y. H. Lee, "On the use of linear programming for dynamic subchannel and bit allocation in multiuser OFDM," in Proc. IEEE Global Communications Conf., San Antonio, TX, 2001, vol. 6, pp. 3648-3652.

[9] P. Viswanath, D. N. C. Tse, and R. Laroia, "Opportunistic beamforming using dumb antennas," IEEE Trans. Inf. Theory, vol. 48, no. 6, pp. 1277-1294, Jun. 2002.

[10] N. Jindal and A. Goldsmith, "Capacity and optimal power allocation for fading broadcast channels with minimum rates," in Proc. IEEE Global Communications Conf., San Antonio, TX, 2001, vol. 2, pp. 1292-1296.

[11] M. C. Hool, J. Tellado, and J. M. Cioffi, "Dual QoS loading algorithms for DMT systems offering CBR and VBR services," in Proc. IEEE Global Communications Conf., Sydney, NSW, Australia, 1998, vol. 1, pp. 25-30.

[12] T. S. Rappaport, Wireless Communications: Principles and Practice. Upper Saddle River, NJ: Prentice-Hall, 2002.

[13] T. M. Cover and J. A. Thomas, Elements of Information Theory. New York: Wiley, 1991.

[14] M. Abramowitz and I. A. Stegun, Handbook of Mathematical Functions With Formulas, Graphs, and Mathematical Tables. New York: Dover, 1972. 9th printing.

[15] R. Baldick, Optimization of Engineering Systems Course Notes, Austin, TX: Univ. Texas. [Online]. Available: http://www.ece.utexas.edu/ rbaldick/

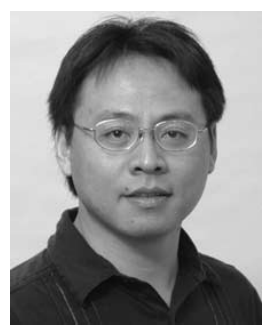

Zukang Shen (S'01) received the B.S. degree in electrical engineering from Tsinghua University, Beijing, China, in 2001, and the M.S. degree in electrical engineering from The University of Texas at Austin, in 2003. He is currently working toward the Ph.D. degree at the Department of Electrical and Computer Engineering, The University of Texas at Austin.

He was awarded the David Bruton, Jr. Graduate Fellowship for the 2004-2005 academic year by the Office of Graduate Studies at The University of Texas at Austin. He also received the UT Austin Texas Telecommunications Engineering Consortium Fellowships for the 2001-2002 and 2003-2004 academic years. His research interests include multicarrier communication systems, resource allocation in multiuser environments, multiple-input multiple-output (MIMO) channel capacity analysis, digital signal processing, and information theory.

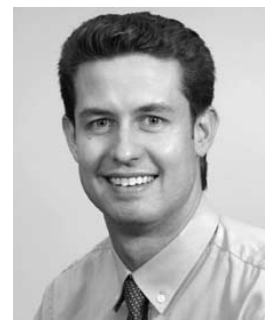

Jeffrey G. Andrews (S'98-M'02) received the B.S. degree in engineering (with high distinction) from the Harvey Mudd College, Claremont, CA, in 1995, and the M.S. and Ph.D. degrees in electrical engineering from Stanford University, Stanford, CA, in 1999 and 2002, respectively.

$\mathrm{He}$ is currently an Assistant Professor at the Department of Electrical and Computer Engineering, The University of Texas at Austin, in the Wireless Networking and Communications Group (WNCG). He helped develop code division multiple access (CDMA) systems as an Engineer at Qualcomm from 1995 to 1997, and has frequently served as a Consultant on communication systems to numerous corporations, startups, and government agencies, including Microsoft, Palm, Ricoh, and NASA. His research interests focus on all areas of multiuser wireless communications, including advanced CDMA systems, orthogonal frequency division multiplexing (OFDM), multiple-input multiple-output (MIMO), and wireless ad hoc networks.

Dr. Andrews serves as an Associate Editor for the IEEE TRANSACTIONS ON Wireless Communications and serves on the Technical Program Committees of ICC and Globecom. 


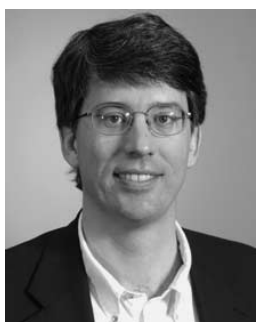

Brian L. Evans (S'88-M'93-SM'97) received the B.S. degree in electrical engineering CS from the Rose-Hulman Institute of Technology, Terre Haute, IN, in 1987, and the M.S. and Ph.D. degrees in electrical engineering from the Georgia Institute of Technology, Atlanta, GA, in 1988 and 1993, respectively.

From 1993 to 1996, he was a Postdoctoral Researcher at the University of California, Berkeley, in design automation for embedded systems. He is the primary architect of the Signals and Systems Pack for Mathematica. He is a Professor of Electrical and Computer Engineering, The University of Texas at Austin. He enjoys conducting research and teaching courses in embedded real-time signal and image processing systems. In signal processing, his research group is focused on the design and real-time software implementation of wireless orthogonal frequency division multiplexing (OFDM) base stations and asymmetric digital subscriber line (ADSL)/very high bit rate digital subscriber line (VDSL) transceivers. His group developed the first ADSL equalization structure that maximizes a measure of bit rate and is realizable in real-time fixed-point software. He has published more than 140 refereed journal and conference papers.

Prof. Evans is an Associate Editor of the IEEE TRANSACTIONS ON SIGNAL Processing, a member of the Design and Implementation of Signal Processing Systems Technical Committee of the IEEE Signal Processing Society, and the recipient of a 1997 National Science Foundation CAREER Award. 